\title{
Article \\ On Subtrees of Fan Graphs, Wheel Graphs, and "Partitions" of Wheel Graphs Under Dynamic Evolution
}

\author{
Yu Yang 1,2, An Wang 3,*, Hua Wang ${ }^{4,5, *}$, Wei-Ting Zhao ${ }^{2}$ and Dao-Qiang Sun ${ }^{2}$ \\ 1 School of Mathematical Sciences, Shanghai Jiao Tong University, Shanghai 200240, China; \\ yangyu@sjtu.edu.cn \\ 2 School of Computer Science, Pingdingshan University, Pingdingshan 467000, China; \\ pdsxyzwt@163.com (W.-T.Z.); aqiang_de@163.com (D.-Q.S.) \\ 3 School of Mathematics and Statistics Science, Pingdingshan University, Pingdingshan 467000, China \\ 4 College of Software, Nankai University, Tianjin 300071, China \\ 5 Department of Mathematical Sciences, Georgia Southern University, Statesboro, GA 30460, USA \\ * Correspondence: kedawangan@163.com (A.W.); hwang@georgiasouthern.edu (H.W.)
}

Received: 18 April 2019; Accepted: 20 May 2019; Published: 24 May 2019

\begin{abstract}
The number of subtrees, or simply the subtree number, is one of the most studied counting-based graph invariants that has applications in many interdisciplinary fields such as phylogenetic reconstruction. Motivated from the study of graph surgeries on evolutionary dynamics, we consider the subtree problems of fan graphs, wheel graphs, and the class of graphs obtained from "partitioning" wheel graphs under dynamic evolution. The enumeration of these subtree numbers is done through the so-called subtree generation functions of graphs. With the enumerative result, we briefly explore the extremal problems in the corresponding class of graphs. Some interesting observations on the behavior of the subtree number are also presented.
\end{abstract}

Keywords: subtree; generating function; fan graph; wheel graph; "partitions" of wheel graph

\section{Introduction}

The study of graph invariants or topological indices has been proven to be of crucial importance in various interdisciplinary topics. Generally, the existing known topological indices could be divided into distance-based, degree-based, or structure-based ones; some of the studies on these three categories are referred to in $[1-4]$ and the references therein. The number of subtrees, or simply the subtree number, is one of the counting-based graph invariants.

Finding and/or enumerating special topological structures or graph patterns has become an important problem due to their applications including, to name a few, frequent subgraphs mining [5], network optimization design [6,7], and local network reliability [8,9]. In particular, the subtree number has also been shown to be correlated to phylogenetic reconstruction [10] and various chemical indices such as the Wiener index (closely correlated with the boiling point of paraffin [3]), the Merrifield-Simmons index, and the Hosoya index [11]. Research results also show that there exists an amazing "negative correlation" between the number of subtrees and the Wiener index [12-17]. Therefore, the subtree number index can indirectly characterize the physical-chemical characteristics of molecules.

Various topics related to the subtree number have been explored over the past years, such as extremal problems $[12,18,19]$, the subtree density problem [20-22], generic chemical structures storage problems [23,24], fault tolerant computing and parallel scheduling [25,26], recognition of substructure [27], 
simplification of the flow networks [28], Markovian queuing systems decomposition [29], and matching problems [30,31].

By using "generating functions", Yan and Yeh [4] presented a linear-time algorithm to evaluate the subtree weight sum of a tree, leading to an algorithmic approach to compute the subtree number of a tree. Following this approach and more recently, Yang et al. [32,33] proposed enumerating algorithms for BC-subtrees of trees, unicyclic, and edge-disjoint bicyclic graphs and computed the subtree number on spiro and polyphenyl hexagonal chains [17]. With structure mapping and weights transferring of cycles, Yang et al. [34] also presented enumerating algorithms for subtrees of hexagonal and phenylene chains. More recently, Chin et al. [35] presented the subtree number of complete graphs, complete bipartite graphs, and theta graphs, as well as the ratio of spanning trees to all subtrees of the above graphs.

Let $K_{1, n}=\left(V\left(K_{1, n}\right), E\left(K_{1, n}\right) ; f, g\right)$ be a weighted star on $n+1$ vertices with center $c_{0}$, vertex weight function $f(v)=y$ for all $v \in V\left(K_{1, n}\right)$, and edge weight function $g(e)=z$ for all $e \in E\left(K_{1, n}\right)$. Suppose the $n$ leaves are labeled, in counterclockwise order, $c_{1}, c_{2}, \ldots, c_{n}$, then the wheel graph $W_{n}$ is obtained from $K_{1, n}$ by adding the edges $\left(c_{i}, c_{i+1}\right)$ for $i=1, \ldots, n$ (here we let $c_{n+1}=c_{1}$ ).

As is well known, star $K_{1, n}$ and wheel graphs $W_{n}$ are very typical network topologies used in designing and implementing communication networks. The wheel graph $W_{n}$ is the planar graph with a chromatic number not greater than 4 . Specifically, the chromatic number of $W_{n}$ is 3 for odd $n$ and 4 for even $n$. Some researchers have tried to apply this property of $W_{n}$ to prove the famous Four Color Theorem [36]. Moreover, $W_{n}$ can also be used in wireless ad hoc networks [37]. Therefore, it is worth studying the new structural characteristics of these two graphs, especially from new perspectives.

Inspired by the significant effect of graph surgeries on evolutionary dynamics in [38], we are motivated to study the subtree number in graphs resulting from graph surgeries. In particular, we will consider a class of graphs that "lie in between" the fan and wheel graphs, called the "partitions" (explanation will be given later) of wheel graphs.

Definition 1. Given the star $K_{1, n}$ on $n+1$ vertices defined above with center vertex $c_{0}$ and $0 \leq j \leq n$, the graph $K_{1, n}^{j}$ is obtained from $K_{1, n}$ by adding the edges $\left(c_{s}, c_{s+1}\right)$ for all $1 \leq s \leq n-1$ except for $s=j, 2 j, 3 j, \ldots$ (see Figure $1 a$ ). If $n=j>0$, we call $K_{1, j}^{j}$ the fan graph on $j+1$ vertices. It is also easy to see that $K_{1,0}^{0}$ is the single vertex $c_{0}$, and $K_{1, n}^{1}$ is the star $K_{1, n}$.

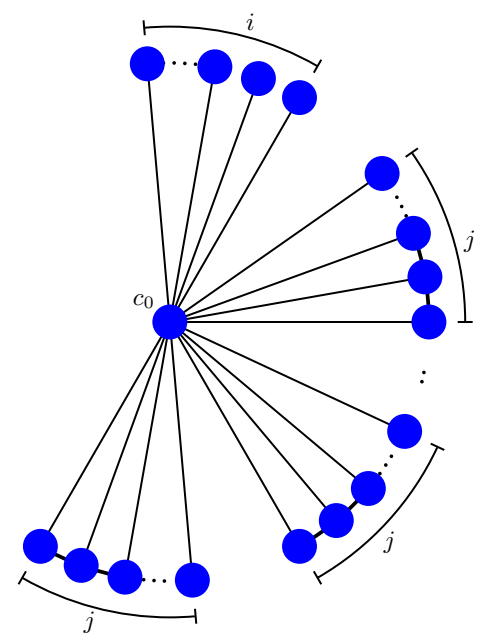

(a) The graph $K_{1, n}^{j}$.

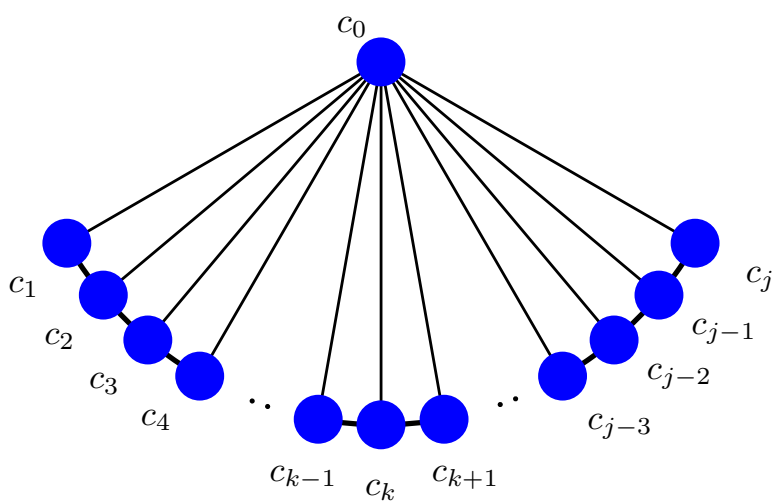

(b) The fan graph $K_{1, j}^{j}$.

Figure 1. The graph $K_{1, n}^{j}$ and the fan graph $K_{1, j}^{j}$. 
From the definitions of the graph $K_{1, n}^{j}$ and the wheel graph $W_{n}$, we see that the graph $K_{1, n}^{j}$ can be vividly described in terms of $W_{n}$ through "partitions" (and deletion of edges $\left\{\left(c_{s}, c_{s+1}\right) \mid s=j, 2 j, 3 j, \ldots\right\}$ ) perspective.

The reason that we consider our graphs as vertex and edge weighted is to use the so-called subtree generating functions. We assume a graph $G=(V(G), E(G) ; f, g)$ to be a weighted graph and define the vertex-weight function $f: V(G) \rightarrow \Re$ and edge-weight function $g: E(G) \rightarrow \Re$, where $\Re$ is the real number. Unless otherwise noted, for a graph $G$, we initialize its vertex weight function $f(v)=y$ for each $v \in V(G)$ and its edge weight function $g(e)=z$ for each $e \in E(G)$ throughout this paper.

The following notations need to be listed before introducing the main tool:

- $G-X$ denotes the graph obtained from $G$ by removing all elements of $X$;

- $S(G)$ (resp. $S(G ; v)$ ) denotes the set of subtrees of $G$ (resp. containing $v$ );

- $S(G ;(a, b))$ denotes the set of subtrees of $G$ containing the edge $(a, b)$;

- $\omega\left(T_{1}\right)$ denotes the weight of subtree $T_{1} \in S(G)$;

- $F(\cdot)$ is the sum of weights of subtrees in $S(\cdot)$;

- $\quad \eta(\cdot)$ is the cardinality (namely the number) of the corresponding $S(\cdot)$ set of subtrees.

We define the weight of a subtree $T_{S}$ of $G$, denoted by $\omega\left(T_{S}\right)$, as the product of the weights of the vertices and edges in $T_{S}$. The generating function of subtrees of $G$, denoted by $F(G ; f, g)$, is the sum of weights of subtrees of $G$. Namely, $F(G ; f, g)=\sum_{T_{1} \in S(G)} \omega\left(T_{1}\right)$. Similarly, we define $F\left(G ; f, g ; v_{i}\right)=\sum_{T_{1} \in S\left(G ; v_{i}\right)} \omega\left(T_{1}\right), F(G ; f, g ;(u, v))=\sum_{T_{1} \in S(G ;(u, v))} \omega\left(T_{1}\right)$.

By substituting each vertex weight $y=1$ and edge weight $z=1$ in these generating functions, we have the corresponding numbers of subtrees under various constraints, i.e., $\eta(T)=$ $F(T ; 1,1), \eta\left(T ; v_{i}\right)=F\left(T ; 1,1 ; v_{i}\right)$, and $\eta(T ;(u, v))=F(T ; 1,1 ;(u, v))$.

We introduce the following lemma, which will frequently be used in our work.

Lemma 1. [4] Let $P_{n}$ be a path on $n$ vertices, with vertex weight function $f(v)=y$ for all $v \in V\left(P_{n}\right)$ and edge weight function $g(e)=z$ for all $e \in E\left(P_{n}\right)$, then $F\left(P_{n} ; f, g\right)=\sum_{t=0}^{n-1}(n-t) y^{t+1} z^{t}$.

In Section 2, we will present the subtree generating functions of $K_{1, n}^{j}(1 \leq j \leq n)$ and the wheel graph $W_{n}$. Through using these generating functions and theoretical analysis, we study the extremal graphs, subtree fitting problems, and subtree density behaviors of these graphs in Section 3. Lastly, in Section 4, we summarize our results and comment on potential topics for future work.

\section{Subtree Generating Functions of $K_{1, N}^{J}(1 \leq J \leq N)$ and Wheel Graph $W_{n}$}

In this section, we will establish the subtree generating functions of $K_{1, n}^{j}(1 \leq j \leq n)$ and wheel graph $W_{n}$ and provide the theoretical background for our computational analysis. We start by studying the subtree problem of $K_{1, n}^{j}(1 \leq j \leq n)$.

\subsection{Subtree Generating Functions and Subtree Numbers of $K_{1, N}^{J}$}

Theorem 1. Let $K_{1, n}^{j}$ be the weighted graph defined as above, and $n, j$ be non-negative integers with $0 \leq j \leq n$ and $n \equiv i(\bmod j)$. Then

$$
F\left(K_{1, n}^{j} ; f, g\right)=F\left(K_{1, j}^{j} ; f, g ; c_{0}\right)^{\frac{n-i}{j}} * y(1+y z)^{i} * y^{-\frac{n-i}{j}}+i y+\frac{n-i}{j} \sum_{t=0}^{j-1}(j-t) y^{t+1} z^{t},
$$


with $F\left(K_{1, j}^{j} ; f, g ; c_{0}\right)=F\left(K_{1, j-1}^{j-1} ; f, g ; c_{0}\right)+\sum_{r=1}^{j} r(y z)^{r} F\left(K_{1, j-r}^{j-r} ; f, g ; c_{0}\right)$, and $F\left(K_{1,0}^{0} ; f, g ; c_{0}\right)=y$, $F\left(K_{1,1}^{1} ; f, g ; c_{0}\right)=y+y^{2} z$

Proof. We consider the subtrees of $K_{1, n}^{j}$ by cases

(i) not containing the center $c_{0}$,

(ii) containing the center $c_{0}$.

From Lemma 1, we have the subtree generating function of case (i) as

$$
\frac{n-i}{j} \sum_{t=0}^{j-1}(j-t) y^{t+1} z^{t}+i y
$$

With the contraction method of [4] and structure analysis, we have the subtree generating function of case (ii) as

$$
F\left(K_{1, j}^{j} ; f, g ; c_{0}\right)^{\frac{n-i}{j}} * y(1+y z)^{i} * y^{-\frac{n-i}{j}}
$$

Denote $e_{j}=\left(c_{0}, c_{j}\right)$ and $\widetilde{e_{j}}=\left(c_{j-1}, c_{j}\right)$, and divide all subtrees for $S\left(K_{1, j}^{j} ; c_{0}\right)$ (see Figure $1 \mathrm{~b}$ ) into four cases $S\left(K_{1, j}^{j} ; c_{0}\right)=\mathcal{S}_{1} \cup \mathcal{S}_{2} \cup \mathcal{S}_{3} \cup \mathcal{S}_{4}$ where

- $\mathcal{S}_{1}$ is the collection of subtrees that contain neither $e_{j}$ nor $\widetilde{e}_{j}$;

- $\mathcal{S}_{2}$ is the collection of subtrees that contain $e_{j}$, but not $\widetilde{e_{j}}$;

- $\mathcal{S}_{3}$ is the collection of subtrees that contain $\widetilde{e}_{j}$, but not $e_{j}$;

- $\mathcal{S}_{4}$ is the collection of subtrees that contain both $e_{j}$ and $\tilde{e}_{j}$.

From the definitions of subtree weight and subtree generating function, we know that:

(a) $\mathcal{S}_{1}=S\left(K_{1, j-1}^{j-1} ; c_{0}\right)$;

(b) $\mathcal{S}_{2}=\left\{T_{1}+e_{j} \mid T_{1} \in \mathcal{S}_{1}\right\}$, where $T_{1}+e_{j}$ are the trees obtained from $T_{1}$ by attaching an edge $e_{j}$ at vertex $c_{0}$;

(c) We can write $\mathcal{S}_{3}$ as

$$
\mathcal{S}_{3}=\left\{T+\left(c_{0}, c_{j-k}\right)+\bigcup_{h=2-k}^{r}\left(c_{j-k-h+1}, c_{j-k-h+2}\right) \mid T \in S\left(K_{1, j-k-r}^{j-k-r} ; c_{0}\right)\right\}
$$

for $k=1,2, \ldots, j-1$ and $r=1,2, \ldots, j-k$;

(d) For each subtree $T_{4} \in \mathcal{S}_{4}$, we know that $T_{4}$ must not contain the edge $\left(c_{0}, c_{j-1}\right)$. Consequently, we can further consider the subtrees that contain edges $\left(c_{0}, c_{j}\right) \bigcup_{r=1}^{k}\left(c_{j-r}, c_{j-r+1}\right)$ but not $\left(c_{j-k-1}, c_{j-k}\right)$ recursively for $k=1,2, \ldots, j-1$.

With (a)-(d), we have

$$
\begin{gathered}
\sum_{T_{1} \in \mathcal{S}_{1}} \omega\left(T_{1}\right)=F\left(K_{1, j-1}^{j-1} ; f, g ; c_{0}\right), \\
\sum_{T_{2} \in \mathcal{S}_{2}} \omega\left(T_{2}\right)=\sum_{T_{1} \in \mathcal{S}_{1}} f\left(c_{j}\right) g\left(e_{j}\right) \omega\left(T_{1}\right)=y z F\left(K_{1, j-1}^{j-1} ; f, g ; c_{0}\right), \\
\sum_{T_{3} \in \mathcal{S}_{3}} \omega\left(T_{3}\right)=\sum_{k=1}^{j-1}\left(\sum_{r=1}^{j-k}\left[\prod_{h=2-k}^{r}\left(f\left(c_{j-k-h+1}\right) g\left(\left(c_{j-k-h+1}, c_{j-k-h+2}\right)\right)\right) f\left(c_{j}\right) g\left(c_{0}, c_{j-k}\right) F\left(K_{1, j-k-r}^{j-k-r} ; f, g ; c_{0}\right)\right]\right) \\
=\sum_{k=1}^{j-1}\left(\sum_{r=1}^{j-k}(y z)^{r+k} F\left(K_{1, j-k-r}^{j-k-r} ; f, g ; c_{0}\right)\right),
\end{gathered}
$$




$$
\begin{aligned}
\sum_{T_{4} \in \mathcal{S}_{4}} \omega\left(T_{4}\right) & =f\left(c_{j}\right) g\left(e_{j}\right) \sum_{k=1}^{j-1}\left(\prod_{r=1}^{k} f\left(c_{j-r}\right) g\left(\left(c_{j-r}, c_{j-r+1}\right)\right)\right) F\left(K_{1, j-k-1}^{j-k-1} ; f, g ; c_{0}\right) \\
& =\sum_{k=1}^{j-1}(y z)^{k+1} F\left(K_{1, j-k-1}^{j-k-1} ; f, g ; c_{0}\right) .
\end{aligned}
$$

Hence, by Equations (5)-(8), we have

$$
\begin{aligned}
F\left(K_{1, j}^{j} ; f, g ; c_{0}\right) & =\sum_{T_{1} \in \mathcal{S}_{1}} \omega\left(T_{1}\right)+\sum_{T_{2} \in \mathcal{S}_{2}} \omega\left(T_{2}\right)+\sum_{T_{3} \in \mathcal{S}_{3}} \omega\left(T_{3}\right)+\sum_{T_{4} \in \mathcal{S}_{4}} \omega\left(T_{4}\right) \\
& =F\left(K_{1, j-1}^{j-1} ; f, g ; c_{0}\right)+\sum_{r=1}^{j} r(y z)^{r} F\left(K_{1, j-r}^{j-r} ; f, g ; c_{0}\right)
\end{aligned}
$$

with $F\left(K_{1,0}^{0} ; f, g ; c_{0}\right)=y, F\left(K_{1,1}^{1} ; f, g ; c_{0}\right)=y+y^{2} z$.

Combining Equations (2), (3), and (9), we have Equation (1) and the theorem follows.

Following similar arguments to Theorem 1, we can also obtain the subtree generating functions of the graphs obtained from identifying the centers of different fan graphs $K_{1, j_{t}}^{j_{t}}(t=1,2, \ldots, l)$. We skip the tedious details for the sake of space.

Theorem 2. Let $K_{1, j_{1}}^{j_{1}}, K_{1, j_{2}}^{j_{2}}, \ldots, K_{1, j_{l}}^{j_{l}}$ be different weighted fan graphs, and suppose there are $n_{j_{t}}$ copies of $K_{1, j_{t}}^{j_{t}}$ for $t=1,2, \ldots, l$. Let $G=\bigcup_{t=1}^{l}\left(K_{1, j_{t}}^{j_{t}}\right)^{n_{j_{t}}}$ be the graph that is constructed by identifying the centers of these $\sum_{t=1}^{l} n_{j_{t}}$ fan graphs with $c_{0}$. Then

$$
F(G ; f, g)=y^{1-\sum_{t=1}^{l} n_{j_{t}}} \prod_{t=1}^{l} F\left(K_{1, j_{t}}^{j_{t}} ; f, g ; c_{0}\right)^{n_{j_{t}}}+\sum_{t=1}^{l}\left(n_{j_{t}} \sum_{r=0}^{j_{t}-1}\left(j_{t}-r\right) y^{r+1} z^{r}\right),
$$

with $F\left(K_{1, j_{t}}^{j_{t}} ; f, g ; c_{0}\right)=F\left(K_{1, j_{t}-1}^{j_{t}-1} ; f, g ; c_{0}\right)+\sum_{r=1}^{j_{t}} r(y z)^{r} F\left(K_{1, j_{t}-r}^{j_{t}-r} ; f, g ; c_{0}\right)$ and $F\left(K_{1,0}^{0} ; f, g ; c_{0}\right)=y$, $F\left(K_{1,1}^{1} ; f, g ; c_{0}\right)=y+y^{2} z$.

Actually, a single fan graph is a special case of the above discussion, so we can further obtain the subtree generating function for the subtrees containing a particular vertex. Again, we skip the similar but technical details.

Theorem 3. Let $K_{1, j}^{j}$ be a weighted fan graph with vertex weigh $f$ and edge weigh $g$ (see Figure $1 b$ ), then

$$
F\left(K_{1, j}^{j} ; f, g ; c_{1}\right)=F\left(K_{1, j}^{j} ; f, g ; c_{0}\right)-F\left(K_{1, j-1}^{j-1} ; f, g ; c_{0}\right)+\sum_{t=0}^{j-1} y^{t+1} z^{t},
$$

with $F\left(K_{1, j}^{j} ; f, g ; c_{0}\right)=F\left(K_{1, j-1}^{j-1} ; f, g ; c_{0}\right)+\sum_{r=1}^{j} r(y z)^{r} F\left(K_{1, j-r}^{j-r} ; f, g ; c_{0}\right)$ and $F\left(K_{1,0}^{0} ; f, g ; c_{0}\right)=y$, $F\left(K_{1,1}^{1} ; f, g ; c_{0}\right)=y+y^{2} z$.

Adding an edge between any two fan graphs (to construct a bigger fan) of a graph will also increase the number of subtrees. Let $G$ be the weighted graph as defined in Theorem 2, and suppose $K_{1, j_{r}}^{j_{r}}$ (with non-center vertices labeled counterclockwise as $c_{j_{1}}^{1}, c_{j_{2}}^{1}, \ldots, c_{j_{r}}^{1}$ ) and $K_{1, j_{s}}^{j_{s}}$ (with non-center vertices labelled clockwise as $c_{j_{1}}^{2}, c_{j_{2}}^{2}, \ldots, c_{j_{s}}^{2}$ ) are the two sub-fan graphs of $G$ with $j_{r} \geq 1$. Define $G^{\prime}=G+$ $\left(c_{j_{r}}^{1}, c_{j_{s}}^{2}\right)$ to be the graph obtained from $G$ by adding one edge $\left(c_{j_{r}}^{1}, c_{j_{s}}^{2}\right)$. Meanwhile, denote $\bar{G}$ the graph of 
$G-\left(\bigcup_{t=1}^{r}\left(c_{0}, c_{j_{t}}^{1}\right) \cup \bigcup_{t=1}^{S}\left(c_{0}, c_{j_{t}}^{2}\right)\right)$ that contains $c_{0}$, and denote $\overline{\mathcal{S}}$ the collection of subtrees in $S\left(K_{1, j_{r}+j_{s}}^{j_{r}+j_{s}} ; c_{0}\right)$ that contain $\left(c_{j_{r}}^{1}, c_{j_{s}}^{2}\right)$. By dividing the subtrees of $G$ and $G^{\prime}$ into two cases of containing center $c_{0}$ or not, with Lemma 1, definitions of subtree weight and subtree generating function, and combining structure analysis, we have the following theorem:

Theorem 4. Let $G$ and $G^{\prime}$ be the weighted graphs defined above. Then,

$$
F\left(G^{\prime} ; f, g\right)=F(G ; f, g)+y^{-1} F\left(\bar{G} ; f, g ; c_{0}\right) \sum_{T \in \overline{\mathcal{S}}} \omega(T)+\sum_{t=0}^{j_{s}} t y^{t+1} z^{t}+j_{s} \sum_{t=j_{s}+1}^{j_{r}} y^{t+1} z^{t}+\sum_{t=j_{r}+1}^{j_{r}+j_{s}-1}\left(j_{r}+j_{s}-t\right) y^{t+1} z^{t} .
$$

By letting $y=z=1$ in the subtree generating functions from the above theorems, we have the corresponding subtree numbers of the various related graphs above.

Corollary 1. The subtree number of $S\left(K_{1, j}^{j}, c_{0}\right)$ is

$$
\eta\left(K_{1, j}^{j} ; c_{0}\right)=\eta\left(K_{1, j-1}^{j-1} ; c_{0}\right)+\sum_{r=1}^{j} r \eta\left(K_{1, j-r}^{j-r} ; c_{0}\right)
$$

with $\eta\left(K_{1,0}^{0} ; c_{0}\right)=1, \eta\left(K_{1,1}^{1} ; c_{0}\right)=2$.

With Corollary 1 , we have the number of subtrees of $K_{1, j}^{j}$ that contain central vertex $c_{0}$ as illustrated in Figure 2.

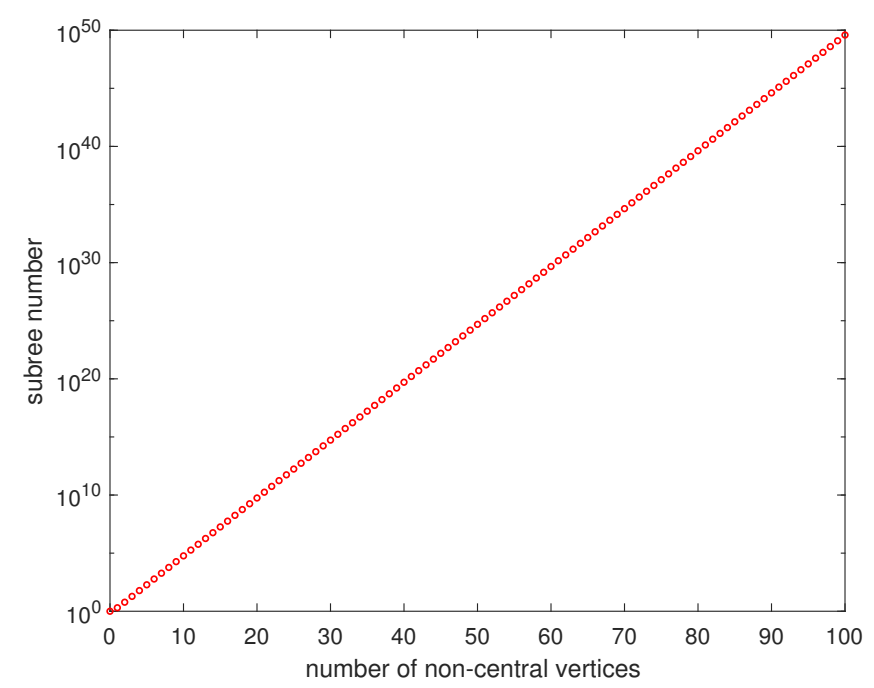

Figure 2. Number of subtrees of $K_{1, j}^{j}$ that contain central vertex $c_{0}$, in semi- $\log (\log -Y)$ coordinates.

Corollary 2. Let $n, j$ be positive integers with $1 \leq j \leq n$, and $i \equiv n(\bmod j)$, then

$$
\eta\left(K_{1, n}^{j}\right)=\frac{(j+1)(n-i)}{2}+i+2^{i} b_{j}^{\frac{n-i}{j}}
$$

with $b_{j}=b_{j-1}+\sum_{r=1}^{j} r b_{j-r}$ and $b_{0}=1, b_{1}=2$.

With Corollary 2, we have the subtree number of $K_{1, n}^{j}$, see details in subsection 3.1. 
Corollary 3. Let $G=\bigcup_{t=1}^{l}\left(K_{1, j_{t}}^{j_{t}}\right)^{n_{j_{t}}, j_{t}}, n_{j_{t}}$ and $l$ be defined in Theorem 2 , then

$$
\eta(G)=\prod_{t=1}^{l} \eta\left(K_{1, j_{t}}^{j_{t}} ; c_{0}\right)^{n_{j_{t}}}+\frac{1}{2} \sum_{t=1}^{l} n_{j_{t}} \times j_{t}\left(j_{t}+1\right)
$$

with $\eta\left(K_{1, j_{t}}^{j_{t}} ; c_{0}\right)=\eta\left(K_{1, j_{t}-1}^{j_{t}-1} ; c_{0}\right)+\sum_{r=1}^{j_{t}} r \eta\left(K_{1, j_{t}-r}^{j_{t}-r} ; c_{0}\right)$ and $\eta\left(K_{1,0}^{0} ; c_{0}\right)=1, \eta\left(K_{1,1}^{1} ; c_{0}\right)=2$.

Let $G_{1}$ be the graph defined in Theorem 2 with $l=4, j_{1}=2, n_{j_{1}}=4 ; j_{2}=3, n_{j_{2}}=3 ; j_{3}=4, n_{j_{3}}=2$; $j_{4}=5, n_{j_{4}}=1$, with Corollary 1 and Corollary 3 , we have $\eta\left(G_{1}\right)=6,048,255,225,665$.

Corollary 4. The number of subtrees of the fan graph $K_{1, j}^{j}$ containing $c_{1}$ is

$$
\eta\left(K_{1, j}^{j} ; c_{1}\right)=\eta\left(K_{1, j}^{j} ; c_{0}\right)-\eta\left(K_{1, j-1}^{j-1} ; c_{0}\right)+j,
$$

with $\eta\left(K_{1, j}^{j} ; c_{0}\right)=\eta\left(K_{1, j-1}^{j-1} ; c_{0}\right)+\sum_{r=1}^{j} r \eta\left(K_{1, j-r}^{j-r} ; c_{0}\right)$ and $\eta\left(K_{1,0}^{0} ; c_{0}\right)=1, \eta\left(K_{1,1}^{1} ; c_{0}\right)=2$.

Similarly, the number of subtrees of $K_{1, j}^{j}$ that contain central vertex $c_{1}$ (see Figure 3) can be obtained from Corollary 4.

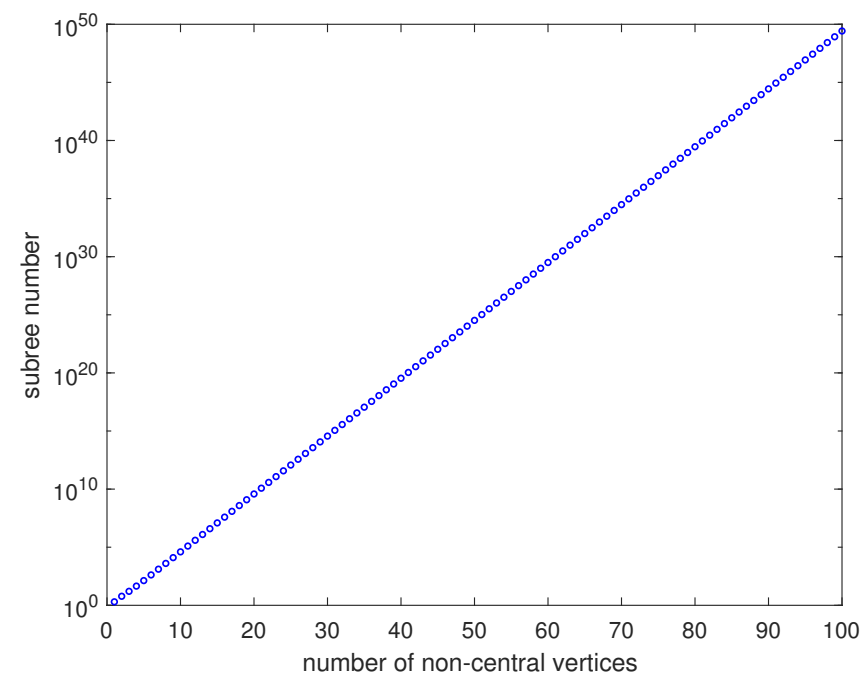

Figure 3. Number of subtrees of $K_{1, j}^{j}$ that contain the vertex $c_{1}$, in semi-log(Log-Y) coordinates.

Corollary 5. Let $G$ be the graph defined in Theorem 2 , and $G^{\prime}$ be a merged graph from $G$ with $j_{r} \geq 1$ and $j_{s} \geq 1$ (Theorem 4), then

$$
\eta\left(G^{\prime}\right)=\eta(G)+\eta\left(\bar{G} ; c_{0}\right) \eta\left(K_{1, j_{r}+j_{s}}^{j_{r}+j_{s}} ; c_{0},\left(c_{j_{r}}^{1}, c_{j_{s}}^{2}\right)\right)+j_{r} j_{s},
$$

where $\eta\left(K_{1, j_{r}+j_{s}}^{j_{r}+j_{s}} ; c_{0},\left(c_{j_{r}}^{1}, c_{j_{s}}^{2}\right)\right)$ is the number of subtrees containing both vertex $c_{0}$ and edge $\left(c_{j_{r}}^{1}, c_{j_{s}}^{2}\right)$.

Let $G, \bar{G}$, and $G^{\prime}$ be the graphs defined in Corollary 5 with $l=2, j_{1}=2, n_{j_{1}}=3 ; j_{2}=3, n_{j_{2}}=2$, $j_{r}=2$ and $j_{s}=3$, with Corollary 1, Corollary 5, and structural analysis, we have $\eta(G)=77,997$, $\eta\left(\bar{G} ; c_{0}\right)=684 \eta\left(K_{1, j_{r}+j_{s}}^{j_{r}+j_{s}} ; c_{0},\left(c_{j_{r}}^{1}, c_{j_{s}}^{2}\right)\right)=75$, and $\eta\left(G^{\prime}\right)=129,303$.

\subsection{Subtree Generating Function and Subtree Number of Wheel Graph $W_{n}$}

Next we consider the subtree generating function of the weighted wheel graph $W_{n}$. 
Theorem 5. Let $W_{n}(n \geq 3)$ be the weighted wheel graph on $n+1$ vertices with vertex weight function $f \equiv y$ and edge weight function $g \equiv z$. Then

$$
\begin{aligned}
F\left(W_{n} ; f, g\right)= & +y z F\left(W_{n-1} ; f, g\right)+(1+2 y z) F\left(K_{1, n-1}^{n-1} ; f, g ; c_{0}\right)-2 y z F\left(K_{1, n-2}^{n-2} ; f, g ; c_{0}\right) \\
& +\sum_{t=1}^{n-1}((1-y z)(n-t)+2 y z) y^{t} z^{t-1}+2 \sum_{k=1}^{n-1}(y z)^{k+1} F\left(K_{1, n-1-k}^{n-1-k} ; f, g ; c_{0}\right) \\
& +\sum_{l=0}^{n-3} \sum_{r=0}^{n-3-l}(y z)^{l+r+3} F\left(K_{1, n-l-r-3}^{n-l-r-3} ; f, g ; c_{0}\right),
\end{aligned}
$$

with $F\left(W_{3} ; f, g\right)=4 y+6 y^{2} z+12 y^{3} z^{2}+16 y^{4} z^{3}, \quad F\left(K_{1, j}^{j} ; f, g ; c_{0}\right)=F\left(K_{1, j-1}^{j-1} ; f, g ; c_{0}\right)+$ $\sum_{r=1}^{j} r(y z)^{r} F\left(K_{1, j-r}^{j-r} ; f, g ; c_{0}\right), F\left(K_{1,0}^{0} ; f, g ; c_{0}\right)=y$, and $F\left(K_{1,1}^{1} ; f, g ; c_{0}\right)=y+y^{2} z$.

Proof. For convenience, we let $e_{t}^{*}=\left(c_{0}, c_{t}\right)$ for $t=1,2, \ldots, n, e_{n}=\left(c_{1}, c_{n}\right)$ and $e_{n-r}=\left(c_{n-r}, c_{n-r+1}\right)$ for $r=1,2, \ldots, n-1$. We also follow the convention that $\bigcup_{r=i}^{j}\left(c_{r}, c_{r+1}\right)=\varnothing$ if $j<i$, and $\sum_{t=i}^{j} b_{t}=0$, if $j<i$.

We first consider the subtrees of $W_{n}(n \geq 3)$ in different cases:

(i) not containing the edge $e_{n}^{*}$,

(ii) containing the edge $e_{n}^{*}$.

The subtrees in case (i) can be further partitioned into four categories. As a result, we have

$$
S\left(W_{n}-e_{n}^{*}\right)=\overline{\mathcal{S}_{1}} \bigcup \overline{\mathcal{S}_{2}} \bigcup \overline{\mathcal{S}_{3}} \bigcup \overline{\mathcal{S}_{4}}
$$

where

- $\quad \overline{\mathcal{S}}_{1}$ is the set of subtrees of $S\left(W_{n}-e_{n}^{*}\right)$ that contain neither $e_{n}$ nor $e_{n-1}$;

- $\quad \overline{\mathcal{S}}_{2}$ is the set of subtrees of $S\left(W_{n}-e_{n}^{*}\right)$ that contain $e_{n-1}$, but not $e_{n}$;

- $\quad \overline{\mathcal{S}}_{3}$ is the set of subtrees of $S\left(W_{n}-e_{n}^{*}\right)$ that contain $e_{n}$ but not $e_{n-1}$;

- $\quad \overline{\mathcal{S}}_{4}$ is the set of subtrees of $S\left(W_{n}-e_{n}^{*}\right)$ that contain both $e_{n}$ and $e_{n-1}$.

From the definition of subtree weight and with structure analysis, we have

$$
\begin{gathered}
\sum_{T \in \overline{\mathcal{S}_{1}}} \omega(T)=y+F\left(K_{1, n-1}^{n-1} ; f, g\right), \\
\sum_{T \in \overline{\mathcal{S}_{2}}} \omega(T)=\sum_{T \in \overline{\mathcal{S}_{3}}} \omega(T)=y z F\left(K_{1, n-1}^{n-1} ; f, g ; c_{1}\right),
\end{gathered}
$$

and

$$
\sum_{T \in \overline{\mathcal{S}_{4}}} \omega(T)=y z\left(F\left(W_{n-1} ; f, g\right)-F\left(K_{1, n-1}^{n-1} ; f, g\right)\right) .
$$

Thus, we have

$$
\begin{aligned}
\sum_{T \in S\left(W_{n}-e_{n}^{*}\right)} \omega(T) & =\sum_{T \in \overline{\mathcal{S}_{1}}} \omega(T)+\sum_{T \in \overline{\mathcal{S}_{2}}} \omega(T)+\sum_{T \in \overline{\mathcal{S}_{3}}} \omega(T)+\sum_{T \in \overline{\mathcal{S}_{4}}} \omega(T) \\
& =y+F\left(K_{1, n-1}^{n-1} ; f, g\right)+y z\left(2 F\left(K_{1, n-1}^{n-1} ; f, g ; c_{1}\right)+F\left(W_{n-1} ; f, g\right)-F\left(K_{1, n-1}^{n-1} ; f, g\right)\right) .
\end{aligned}
$$

Similarly, for case (ii), we have

$$
S\left(W_{n} ; e_{n}^{*}\right)=\mathcal{S}_{1} \bigcup \mathcal{S}_{2} \bigcup \mathcal{S}_{3} \bigcup \mathcal{S}_{4}
$$


where

- $\mathcal{S}_{1}$ is the set of subtrees in $S\left(W_{n} ; e_{n}^{*}\right)$ that contain neither $e_{n}$ nor $e_{n-1}$;

- $\mathcal{S}_{2}$ is the set of subtrees in $S\left(W_{n} ; e_{n}^{*}\right)$ that contain $e_{n}$, but not $e_{n-1}$;

- $\mathcal{S}_{3}$ is the set of subtrees in $S\left(W_{n} ; e_{n}^{*}\right)$ that contain $e_{n-1}$ but not $e_{n}$;

- $\mathcal{S}_{4}$ is the set of subtrees in $S\left(W_{n} ; e_{n}^{*}\right)$ that contain both $e_{n}$ and $e_{n-1}$.

Analyzing each case, we have:

(a) $\mathcal{S}_{1}=\left\{T+\left(c_{0}, c_{n}\right) \mid T \in S\left(K_{1, n-1}^{n-1} ; c_{0}\right)\right\}$, where $T+\left(c_{0}, c_{n}\right)$ are the trees obtained from $T\left(\in S\left(K_{1, n-1}^{n-1} ; c_{0}\right)\right)$ by attaching the edge $\left(c_{n}, c_{0}\right)$ at vertex $c_{0}$;

(b) $\mathcal{S}_{2}=\left\{T+e_{n}^{*}+\left(c_{1}, c_{n}\right)+\bigcup_{r=1}^{k-1}\left(c_{r}, c_{r+1}\right) \mid T \in S\left(\widetilde{K}_{1, n-1-k}^{n-1-k} c_{0}\right)\right\}$, where $\widetilde{K}_{1, n-1-k}^{n-1-k}(k=1,2, \ldots, n-1)$ is the graph of $W_{n}-\left(e_{k} \cup e_{n-1} \cup e_{n}^{*} \bigcup_{r=1}^{k} e_{r}^{*}\right)$ that contains $c_{0}$, and obviously, $\widetilde{K}_{1, n-1-k}^{n-1-k} \cong K_{1, n-1-k}^{n-1-k}$;

(c) $\mathcal{S}_{3}=\left\{T+e_{n}^{*}+\bigcup_{r=1}^{k}\left(c_{n-r}, c_{n-r+1}\right) \mid T \in S\left(K_{1, n-1-k}^{n-1-k} ; c_{0}\right)\right\}$ for $k=1,2, \ldots, n-1$;

(d) In a similar manner as (b) and (c), use variables $l$ and $r$ to count the number of edges trailing off $\left(c_{n-1}, c_{n}\right)$ and $\left(c_{1}, c_{n}\right)$, respectively, then the subtrees in $\mathcal{S}_{4}$ are indexed by these two variables, which count extra edges that are on the two sides of the $T$ shape containing the 3 edges required by $\mathcal{S}_{4}$. With (a)-(d), we have

$$
\begin{aligned}
& \sum_{T_{1} \in \mathcal{S}_{1}} \omega\left(T_{1}\right)=\sum_{T \in S\left(K_{1, n-1}^{n-1} ; c_{0}\right)} f\left(c_{n}\right) g\left(e_{n}^{*}\right) \omega(T)=y z F\left(K_{1, n-1}^{n-1} ; f, g ; c_{0}\right), \\
& \sum_{T_{2} \in \mathcal{S}_{2}} \omega\left(T_{2}\right)=\sum_{T_{3} \in \mathcal{S}_{3}} \omega\left(T_{3}\right)=f\left(c_{n}\right) g\left(e_{n}^{*}\right) \sum_{k=1}^{n-1} \prod_{r=1}^{k}\left(g\left(e_{n-r}\right) f\left(c_{n-r}\right)\right) F\left(K_{1, n-1-k^{\prime}}^{n-1-k}, g ; c_{0}\right) \\
& =y z \sum_{k=1}^{n-1}(y z)^{k} F\left(K_{1, n-1-k}^{n-1-k} ; f, g ; c_{0}\right) \text {, } \\
& \sum_{T_{4} \in \mathcal{S}_{4}} \omega\left(T_{4}\right)=\sum_{l=0}^{n-3} \sum_{r=0}^{n-3-l}(y z)^{l+r+3} F\left(K_{1, n-l-r-3}^{n-l-r-3} ; f, g ; c_{0}\right) .
\end{aligned}
$$

Now with Equations (23)-(25), we have

$$
\begin{aligned}
\sum_{T \in S\left(W_{n} ;\left(c_{0}, c_{n}\right)\right)} \omega(T)= & \sum_{T_{1} \in \mathcal{S}_{1}} \omega\left(T_{1}\right)+\sum_{T_{2} \in \mathcal{S}_{2}} \omega\left(T_{2}\right)+\sum_{T_{3} \in \mathcal{S}_{3}} \omega\left(T_{3}\right)+\sum_{T_{4} \in \mathcal{S}_{4}} \omega\left(T_{4}\right) \\
= & y z F\left(K_{1, n-1}^{n-1} ; f, g ; c_{0}\right)+2 \sum_{k=1}^{n-1}(y z)^{k+1} F\left(K_{1, n-1-k}^{n-1-k} ; f, g ; c_{0}\right) \\
& +\sum_{l=0}^{n-3} \sum_{r=0}^{n-3-l}(y z)^{l+r+3} F\left(K_{1, n-l-r-3}^{n-l-r-3} ; f, g ; c_{0}\right) .
\end{aligned}
$$

By Theorem 1, Theorem 3, and Equations (22) and (26), we have

$$
\begin{aligned}
F\left(W_{n} ; f, g\right)= & y+y z F\left(W_{n-1} ; f, g\right)+(1+2 y z) F\left(K_{1, n-1}^{n-1} ; f, g ; c_{0}\right)-2 y z F\left(K_{1, n-2}^{n-2} ; f, g ; c_{0}\right) \\
& +\sum_{t=1}^{n-1}((1-y z)(n-t)+2 y z) y^{t} z^{t-1}+2 \sum_{k=1}^{n-1}(y z)^{k+1} F\left(K_{1, n-1-k}^{n-1-k} ; f, g ; c_{0}\right) \\
& +\sum_{l=0}^{n-3} \sum_{r=0}^{n-3-l}(y z)^{l+r+3} F\left(K_{1, n-l-r-3}^{n-l-r-3} ; f, g ; c_{0}\right) .
\end{aligned}
$$

Note that $W_{2}$ is not a wheel graph. With Theorem 1 and Lemma 1 , we have

$$
\sum_{T \in S\left(W_{2}\right)} \omega(T)=3 y+4 y^{2} z+5 y^{3} z^{2} .
$$


Now from Equations (27) and (28), we have

$$
F\left(W_{3} ; f, g\right)=4 y+6 y^{2} z+12 y^{3} z^{2}+16 y^{4} z^{3} .
$$

The subtree generating function of $W_{n}$ now follows from Equations (27) and (29).

Letting $y=z=1$ in Equation (18), we have the following corollary.

Corollary 6. The subtree number of $W_{n}(n \geq 3)$ is

$$
\eta\left(W_{n}\right)=\eta\left(W_{n-1}\right)+3 \eta\left(K_{1, n-1}^{n-1} ; c_{0}\right)+2 n-1+2 \sum_{k=2}^{n-1} \eta\left(K_{1, n-1-k}^{n-1-k} ; c_{0}\right)+\sum_{l=0}^{n-3} \sum_{r=0}^{n-3-l} \eta\left(K_{1, n-l-r-3}^{n-l-r-3} ; c_{0}\right),
$$

with $\eta\left(W_{3}\right)=38, \eta\left(K_{1, j}^{j} ; c_{0}\right)=\eta\left(K_{1, j-1}^{j-1} ; c_{0}\right)+\sum_{r=1}^{j} r \eta\left(K_{1, j-r}^{j-r} ; c_{0}\right), \eta\left(K_{1,0}^{0} ; c_{0}\right)=1$ and $\eta\left(K_{1,1}^{1} ; c_{0}\right)=2$.

With Corollary 6, the subtree numbers of $W_{n}(n=3,4, \ldots, 50)$ are shown in Table 1.

Table 1. The subtree numbers of wheel graph $W_{n}(n=3,4, \ldots, 50)$.

\begin{tabular}{cccccc}
\hline $\boldsymbol{n}$ & $\boldsymbol{\eta}\left(\boldsymbol{W}_{\boldsymbol{n}}\right)$ & $\boldsymbol{n}$ & $\eta\left(\boldsymbol{W}_{\boldsymbol{n}}\right)$ & $\boldsymbol{n}$ & $\eta\left(\boldsymbol{W}_{\boldsymbol{n}}\right)$ \\
\hline 3 & 38 & 19 & $2,899,980,984$ & 35 & $269,604,917,347,967,886$ \\
4 & 112 & 20 & $9,128,846,611$ & 36 & $848,689,059,340,934,448$ \\
5 & 332 & 21 & $28,736,686,630$ & 37 & $2,671,587,471,512,527,895$ \\
6 & 1007 & 22 & $90,460,187,232$ & 38 & $8,409,887,625,375,274,755$ \\
7 & 3110 & 23 & $284,759,535,167$ & 39 & $26,473,477,146,304,448,341$ \\
8 & 9704 & 24 & $896,394,265,075$ & 40 & $83,335,833,180,604,495,475$ \\
9 & 30,431 & 25 & $2,821,758,641,457$ & 41 & $262,332,788,908,879,910,034$ \\
10 & 95,643 & 26 & $8,882,611,305,147$ & 42 & $825,797,133,240,010,600,373$ \\
11 & 300,885 & 27 & $27,961,563,560,618$ & 43 & $2,599,525,999,414,007,165,103$ \\
12 & 946,923 & 28 & $88,020,178,967,761$ & 44 & $8,183,045,386,844,876,767,480$ \\
13 & $2,980,538$ & 29 & $277,078,636,493,555$ & 45 & $25,759,400,682,377,496,173,050$ \\
14 & $9,382,101$ & 30 & $872,215,572,630,716$ & 46 & $81,087,992,568,389,361,552,840$ \\
15 & $29,533,519$ & 31 & $2,745,646,560,009,062$ & 47 & $255,256,813,613,269,834,457,576$ \\
16 & $92,968,088$ & 32 & $8,643,018,158,636,696$ & 48 & $803,522,677,430,288,749,342,627$ \\
17 & $292,653,642$ & 33 & $27,207,348,527,149,292$ & 49 & $2,529,408,261,449,734,855,548,318$ \\
18 & $921,243,536$ & 34 & $85,645,986,192,695,055$ & 50 & $7,962,321,827,121,343,008,620,568$ \\
\hline
\end{tabular}

As a matter of fact, with the generating function and further structural and theoretical analysis, we can also solve the subtree generation computing problems for the following more generalized types of graphs; here we skip the similar but technical details.

(i) Graph $G=\bigcup_{i=1}^{l} F_{i, n_{i}}^{t_{i}}$ is constructed by identifying the center $c_{0}^{i}$ of $l$ graphs $F_{i, n_{i}}^{t_{i}}(i=1,2, \ldots, l)$ to $c_{0}$, where $\overline{F_{i, n_{i}}^{t_{i}}}\left(n_{i} \geq 1,2 \leq t_{i} \leq n_{i}\right)$ is the graph constructed from a vertex $c_{0}^{i}$ and a path $P_{c_{1}^{i} c_{n_{i}}^{i}}=$ $c_{1}^{i} c_{2}^{i} \cdots c_{n_{i}}^{i}$ by connecting $c_{0}^{i}$ with $c_{1}^{i}, c_{n_{i}}^{i}$, and any other arbitrary $t_{i}-2$ vertices on the path $P_{c_{1}^{i}} c_{n_{i}}^{i}$; for the special case $n_{i}=1$, the graph $F_{i, n_{i}}^{t_{i}}$ is a path $c_{0}^{i} c_{1}^{i}$ on vertices $c_{0}^{i}$ and $c_{1}^{i}$.

(ii) Graph $G=W_{n}-\bigcup_{t=1}^{l}\left(c_{0}, c_{j_{t}}\right)\left(1 \leq l, j_{t} \leq n\right)$, namely the graph obtained from wheel $W_{n}$ by deleting random $l$ different edges $\left(c_{0}, c_{j_{t}}\right)(t=1,2, \ldots, l)$ (each $j_{t}$ is different the others).

\section{Behaviors of $K_{1, N}^{J}(1 \leq J \leq N)$ and $W_{n}$ in Terms of Subtrees}

With the subtree generating functions established, we can now analyze the behaviors of the graphs $K_{1, n}^{j}(1 \leq j \leq n)$ and $W_{n}$ in terms their subtree numbers and other related properties. 


\subsection{Subtree Numbers and $K_{1, N}^{J}$}

We start with the subtree numbers of the graphs $K_{1, n}^{j}$ for different $n$ and $j$. First, we take a quick look at the extremal problems.

Proposition 1. Among all $K_{1, n}^{j}(1 \leq j \leq n)$ :

- the graph $K_{1, n}^{1}$ has $2^{n}+n$ subtrees, fewer than any other $K_{1, n}^{j}(j \neq 1)$; and

- the graph $K_{1, n}^{n}$ has $\frac{n(n+1)}{2}+b_{n}$ subtrees (where $b_{n}=b_{n-1}+\sum_{r=1}^{n} r b_{n-r}$ and $b_{0}=1, b_{1}=2$ ), more than any other $K_{1, n}^{j}(j \neq n)$.

Proof. Note that adding an edge to a graph will strictly increase the subtree number. The extremal structures $K_{1, n}^{1}$ and $K_{1, n}^{n}$ then follow immediately from the fact that the former is a subgraph of any $K_{1, n}^{j}$ and the latter contains any $K_{1, n}^{j}$ as a subgraph.

We are also interested in knowing which $K_{1, n}^{j}(2 \leq j \leq n-1)$ has the second- or third-largest subtree number. To examine this, we also explore how the subtree numbers of the graphs $K_{1, n}^{j}$ behave. With Corollary 2 and Matlab computing (see Appendix A for more details), we obtain the subtree number behaviors of $K_{1, n}^{j}$ as shown in Figure 4 .

It seems from Figure $4 \mathrm{a}, \mathrm{b}$ that among all $K_{1, n}^{j}(1 \leq j \leq n-1)$ :

- $\quad K_{1, n}^{n-1}$ has the second-largest subtree number; and

- $\quad K_{1, n}^{n-2}$ has the third-largest subtree number for odd (or sufficiently large) $n$.

- $\quad$ the subtree number $K_{1, n}^{j}$ increment trend meets exponential growth when $j \geq\left\lceil\frac{n+1}{2}\right\rceil$.
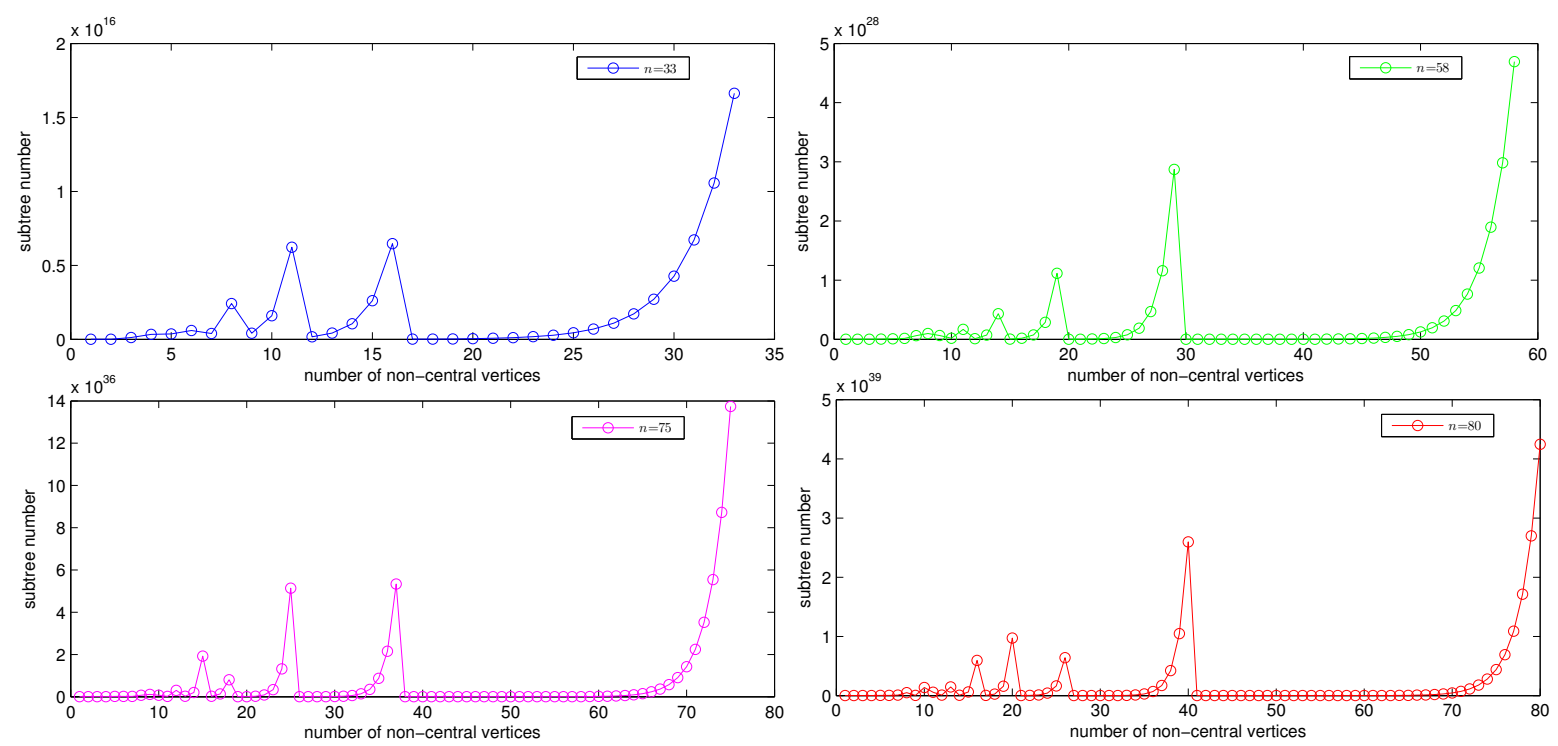

(a) Subtree numbers of $K_{1, n}^{j}$ with $n=33,58,75,80, j$ from 1 to $n$, in Cartesian coordinates.

Figure 4. Cont. 

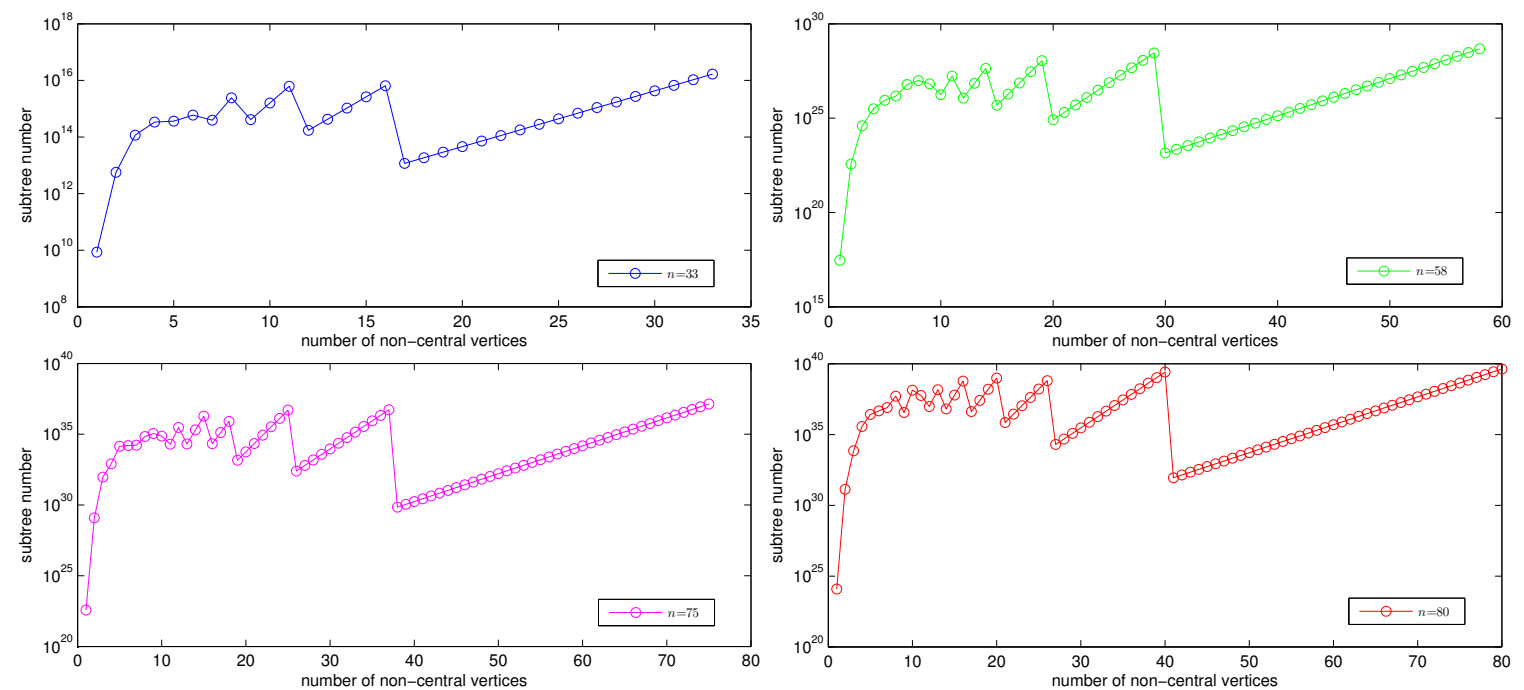

(b) Subtree numbers of $K_{1, n}^{j}$ with $n=33,58,75,80, j$ from 1 to $n$, in semi-log(Log-Y) coordinates.

Figure 4. Subtree numbers of $K_{1, n}^{j}$ with $n=33,58,75,80, j$ from 1 to $n$.

In general, it is not difficult to show the following.

Proposition 2. Suppose $n>>k$ (i.e., $n$ is much larger than $k$ ), then among all $K_{1, n}^{j}(1 \leq j \leq n)$, the graph $K_{1, n}^{n-k}$ has the $(k+1)$-th largest subtree number.

The proof follows from adjusting the "sizes" of the two sub-fan graphs of $K_{1, n}^{j}$. We skip the details here. The same argument can also show the monotonic behavior for large enough $j$.

Proposition 3. If $j>\frac{n}{2}$, then $K_{1, n}^{j+1}$ has a larger subtree number than $K_{1, n}^{j}$.

Understanding the specific behavior of $K_{1, n}^{j}$ for general $j$ in terms of the subtree number seems to be an interesting and nontrivial problem.

Similarly, from Corollary 6, we can obtain the subtree numbers of wheel graph $W_{n}(n \geq 3)$, as already shown in Table 1. On the other hand, experimental observation shows that the subtree numbers of $W_{n}(n \geq 3)$ increase very fast and this growth trend seems to fit the linear regression model after doing the logarithmic transformation. Through implementing the linear regression in the MATLAB software for data of the number of subtrees of $W_{n}$ from $n=3$ to 602 , the appropriate formula for the subtree number of $W_{n}$ can be stated as

$$
\eta\left(W_{n}\right) \approx \exp (0.0126+1.1466 n) .
$$

As $\exp 0.0126 \approx 1$, Equation (31) can be rewritten as

$$
\eta\left(W_{n}\right) \approx \exp 1.1466 n \text {. }
$$

With Corollary 6, Equation (32), and logarithmic transformation for each subtree number of $W_{n}$ and fitted value, we can obtain the subtree number trend of $W_{n}(n \geq 3)$, as illustrated in Figure 5 where the original and fitted data are respectively marked in red and blue. We believe Equation (32) can be proved or disproved through traditional analytic combinatorial approaches, but we will not pursue the technical analysis here. 


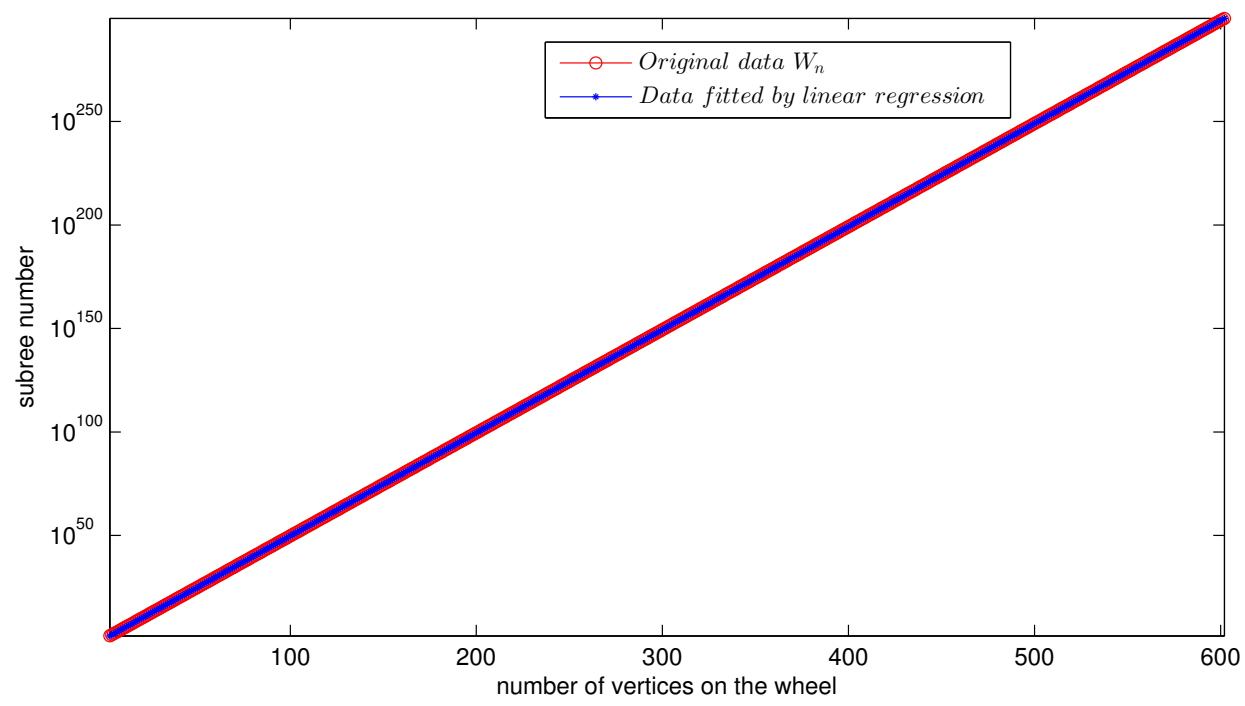

Figure 5. Subtree number trend of wheel graph $W_{n}(n=3,4, \ldots, 602)$, in semi-log $(\log -Y)$ coordinates.

Our work can also be easily applied to more general "partitions" of wheel graphs instead of just the $K_{1, n}^{j}$ s. To illustrate the observations, we introduce some simple notations. Given a positive integer $n$ and partition $\pi=\left(\pi_{1}, \pi_{2}, \ldots, \pi_{k}\right)$ of $n$ (with $\pi_{1} \geq \pi_{2} \geq \ldots \geq \pi_{k}$ and $n=\sum_{i=1}^{k} \pi_{i}$ ), the graph $K_{\pi}$ is obtained from $K_{1, n}$ by adding edges $\left(c_{j}, c_{j+1}\right)$ for all $1 \leq j \leq n-1$ except for $j=\sum_{i=1}^{s} \pi_{i}$, for $s=1,2, \ldots, k$. It seems that the subtree numbers of $K_{\pi} s$ coincide with some sort of ordering of the partitions $\pi$ of $n$. In general, larger and fewer parts in $\pi$ results in more subtrees in $K_{\pi}$. More precise statements along this line requires further study.

\subsection{Subtree Densities of $K_{1, N}^{J}$ and $W_{n}$}

The subtree generating function can be used to provide more information than just the number of subtrees. For instance, using the subtree generating function, one can easily obtain the total number of vertices in all subtrees, from which we have the average subtree order in a given graph. The ratio of the average subtree order and the order of the original graph is called the subtree density of the graph. We present the formal subtree density definition as follows:

Definition 2 ([20]). Suppose $G$ is a graph with $n$ vertices, then $\mu(G)=\frac{1}{k} \sum_{i=1}^{k} n_{i}$ is the average order of the subtrees of $G$, where $n_{1}, n_{2}, \ldots, n_{k}$ are the orders of all of $G$ 's $k$ non-empty subtrees, and the subtree density of $G$ is defined as $D(G)=\frac{\mu(G)}{n}$.

It is essentially the probability that a vertex chosen at random from $G$ will belong to a randomly chosen subtree of $G$. Some of the work related to subtree densities can be found in [20,21,39].

With Theorem 1, Theorem 5, and substituting $z=1$, we could obtain the vertex generating function of subtrees of $K_{1, n}^{j}$ and $W_{n}$, respectively, i.e., $F\left(K_{1, n}^{j} ; y, 1\right)$ and $\left.F\left(W_{n} ; y, 1\right)\right)$. The subtree density of $K_{1, n}^{j}$ and $W_{n}$ is simply

$$
D\left(G^{*}\right)=\frac{\left.\frac{\partial F\left(G^{*} ; y, 1\right)}{\partial y}\right|_{y=1}}{F\left(G^{*} ; 1,1\right) * n\left(G^{*}\right)},
$$

where $n\left(G^{*}\right)$ denotes the vertex number of $G^{*}$, and $G^{*}$ can be $K_{1, n}^{j}$ or $W_{n}$.

We will now apply this to find the subtree densities of $K_{1, n}^{j}$ and $W_{n}$. 
Clearly, the vertex number of $K_{1, n}^{j}$ and $W_{n}$ is $n+1$, namely

$$
n\left(K_{1, n}^{j}\right)=n\left(W_{n}\right)=n+1 .
$$

With Theorem 1 and letting $z=1$ in Equation (1), we obtain the vertex generating function of subtrees of $K_{1, n}^{j}(1 \leq j \leq n)$.

$$
F\left(K_{1, n}^{j} ; f, 1\right)=(1+y)^{i} y^{1-\frac{n-i}{j}} * F\left(K_{1, j}^{j} ; f, 1 ; c_{0}\right)^{\frac{n-i}{j}}+i y+\frac{n-i}{j} \sum_{t=0}^{j-1}(j-t) y^{t+1}
$$

with $i \equiv n(\bmod j), F\left(K_{1, j}^{j} ; f, 1 ; c_{0}\right)=F\left(K_{1, j-1}^{j-1} ; f, 1 ; c_{0}\right)+\sum_{r=1}^{j} r y^{r} F\left(K_{1, j-r}^{j-r} ; f, 1 ; c_{0}\right)$ and $F\left(K_{1,0}^{0} ; f, 1 ; c_{0}\right)=$ $y, F\left(K_{1,1}^{1} ; f, 1 ; c_{0}\right)=y+y^{2}$.

Similarly, letting $z=1$ in Equation (18), we obtain the vertex generating function of subtrees of $W_{n}$.

$$
\begin{aligned}
F\left(W_{n} ; f, 1\right)= & \left.y+y F\left(W_{n-1} ; f, 1\right)+(1+2 y) F\left(K_{1, n-1}^{n-1} ; f, 1 ; c_{0}\right)-2 y F\left(K_{1, n-2}^{n-2} ;\right) f, 1 ; c_{0}\right) \\
& +\sum_{t=1}^{n-1}((1-y)(n-t)+2 y) y^{t}+2 \sum_{k=1}^{n-1} y^{k+1} F\left(K_{1, n-1-k}^{n-1-k} ; f, 1 ; c_{0}\right) \\
& +\sum_{l=0}^{n-3} \sum_{r=0}^{n-3-l} y^{l+r+3} F\left(K_{1, n-l-r-3}^{n-l-r-3} ; f, 1 ; c_{0}\right)
\end{aligned}
$$

with $F\left(W_{3} ; f, 1\right)=4 y+6 y^{2}+12 y^{3}+16 y^{4}, F\left(K_{1, j}^{j} ; f, 1 ; c_{0}\right)=F\left(K_{1, j-1}^{j-1} ; f, 1 ; c_{0}\right)+\sum_{r=1}^{j} r y^{r} F\left(K_{1, j-r}^{j-r} ; f, 1 ; c_{0}\right)$ and $F\left(K_{1,0}^{0} ; f, 1 ; c_{0}\right)=y, F\left(K_{1,1}^{1} ; f, 1 ; c_{0}\right)=y+y^{2}$.

From Equations (33)-(35), we can obtain the subtree densities of $K_{1, n}^{j}(1 \leq j \leq n)$ (plotted in Figure 6); related data can be found in Table 2. Similarly, from Equations (33), (34), and (36), we have subtree densities $W_{n}$ plotted in Figure 7; related data are listed in Table 3.

From Table 2 and Figure 6, it appears that $K_{1,22}^{1}$ and $K_{1,22}^{12}$ have the smallest and second-smallest subtree densities, respectively. $K_{1,22}^{j}(12 \leq j \leq 22)$ grows linearly with the increase of $j$. Moreover, from Table 3 and Figure 7 , we see that $W_{n}(3 \leq n \leq 24)$ increases first in the interval $[3,8]$ and reaches the maximum value when $n=8$, and then decreases gradually in the interval $[9,24]$ and approximates the limit value of 0.8135 .

Similarly, as studied in paper [35], we can also discuss the ratio of spanning trees to all subtrees and spanning tree densities of $K_{1, n}^{j}$ and $W_{n}$. We skip the details here.

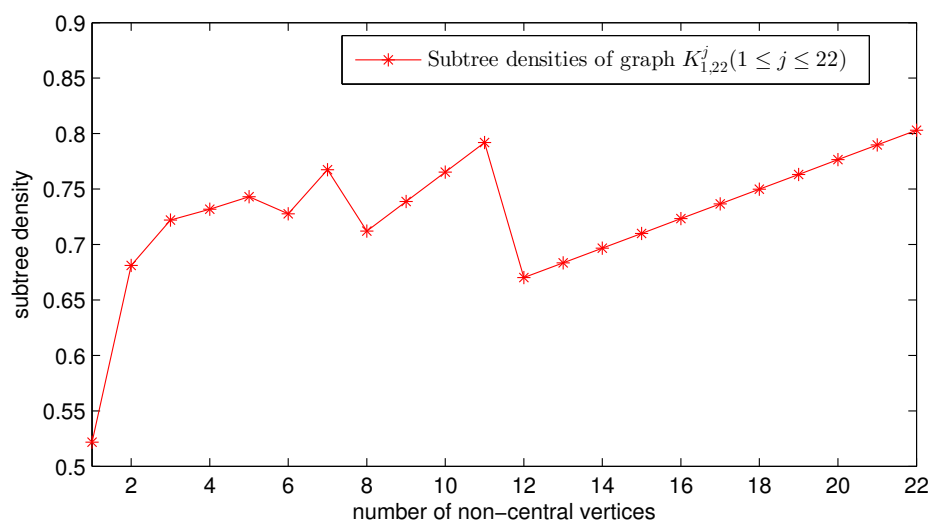

Figure 6. Subtree densities of the graphs $K_{1,22}^{j}(1 \leq j \leq 22)$. 
Table 2. Related data for $K_{1, n}^{j}$, with $n=22$ and $j=1,2, \ldots, 22$.

\begin{tabular}{|c|c|c|c|}
\hline$j$ & $P_{y}\left(K_{1, n}^{j}\right)$ & $\eta\left(K_{1, n}^{j}\right)$ & $D\left(K_{1, n}^{j}\right)$ \\
\hline 1 & $50,331,670$ & $4,194,326$ & 0.521736621869847 \\
\hline 2 & $5,683,820,588$ & $362,797,089$ & 0.681159363604678 \\
\hline 3 & $29,685,950,982$ & $1,787,743,521$ & 0.721967947748206 \\
\hline 4 & $52,358,400,102$ & $3,110,400,052$ & 0.731884047161090 \\
\hline 5 & $87,226,395,622$ & $5,103,959,426$ & 0.743041170007376 \\
\hline 6 & $56,400,430,972$ & $3,370,318,067$ & 0.727584934777173 \\
\hline 7 & $231,968,014,120$ & $13,141,451,319$ & 0.767462100378713 \\
\hline 8 & $36,440,865,014$ & $2,224,820,302$ & 0.712140856484914 \\
\hline 9 & $93,645,742,414$ & $5,511,577,694$ & 0.738727501280144 \\
\hline 10 & $240,339,417,442$ & $13,653,922,612$ & 0.765314128820302 \\
\hline 11 & $616,080,713,876$ & $33,825,095188$ & 0.791900742502907 \\
\hline 12 & $9,137,267,062$ & $592,843,864$ & 0.670113169892776 \\
\hline 13 & $14,666,890,448$ & $933,106,276$ & 0.683406494463958 \\
\hline 14 & $23,533,982,520$ & $1,468,662,129$ & 0.696699813456106 \\
\hline 15 & $37,748,062,639$ & $2,311,599,999$ & 0.709993128330647 \\
\hline 16 & $60,525,953,078$ & $3,638,341,646$ & 0.723286440182849 \\
\hline 17 & $97,015,668,926$ & $5,726,566,014$ & 0.736579749833999 \\
\hline 18 & $155,453,553,144$ & $9,013,325,743$ & 0.749873057893726 \\
\hline 19 & $249,013,516,065$ & $14,186,519,625$ & 0.763166364810336 \\
\hline 20 & $398,761,664,190$ & $22,328,865,632$ & 0.776459670910826 \\
\hline 21 & $638,376,020,719$ & $35,144,507,194$ & 0.789752976431952 \\
\hline 22 & $1,021,684,176,864$ & $55,315,680,041$ & 0.803046281543951 \\
\hline
\end{tabular}

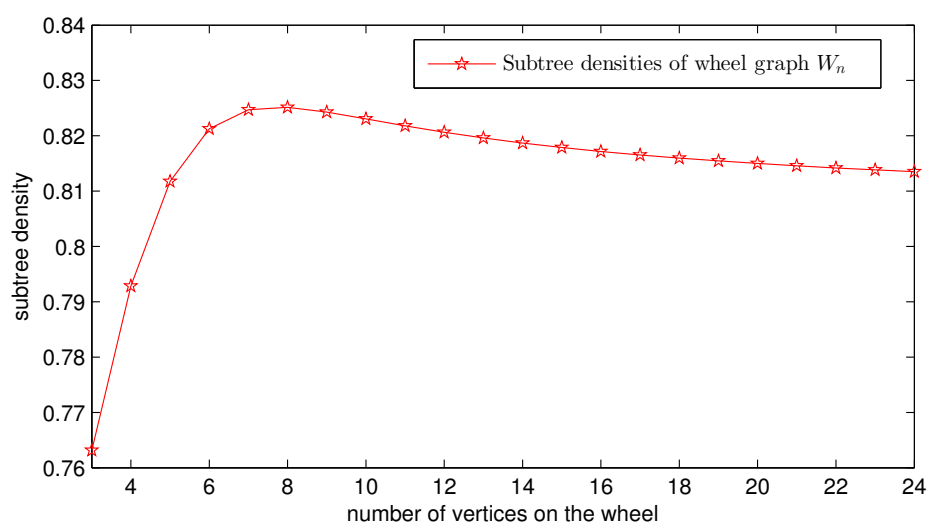

Figure 7. Subtree densities of wheel graph $W_{n}(3 \leq n \leq 24)$.

Table 3. Related data for wheel graph $W_{n}(n=3,4 \ldots, 24)$.

\begin{tabular}{cccccc}
\hline $\boldsymbol{k}$ & $\boldsymbol{P}_{\boldsymbol{y}}\left(\boldsymbol{W}_{\boldsymbol{k}}\right)$ & $\boldsymbol{D}\left(\boldsymbol{W}_{\boldsymbol{k}}\right)$ & $\boldsymbol{k}$ & $\boldsymbol{P}_{\boldsymbol{y}}\left(\boldsymbol{W}_{\boldsymbol{k}}\right)$ & $\boldsymbol{D}\left(\boldsymbol{W}_{\boldsymbol{k}}\right)$ \\
\hline 3 & 116 & 0.7631578947368421 & 14 & $115,215,423$ & 0.8186895664414613 \\
4 & 444 & 0.7928571428571428 & 15 & $386,480,089$ & 0.8178844370865525 \\
5 & 1617 & 0.8117469879518072 & 16 & $1,291,505,336$ & 0.8171718247840813 \\
6 & 5789 & 0.8212512413108243 & 17 & $4,301,328,493$ & 0.8165375712479791 \\
7 & 20,519 & 0.8247186495176849 & 18 & $14,282,430,812$ & 0.8159697793983819 \\
8 & 72,064 & 0.8251351103783091 & 19 & $47,296,291,958$ & 0.815458656779937 \\
9 & 250,841 & 0.8242943051493543 & 20 & $156,239,476,051$ & 0.8149961728004785 \\
10 & 865,923 & 0.8230636087039588 & 21 & $514,980,557,554$ & 0.8145757185906111 \\
11 & $2,967,219$ & 0.8218031806171793 & 22 & $1,693,994,724,188$ & 0.8141918205521789 \\
12 & $10,102,071$ & 0.8206394655271703 & 23 & $5,561,968,202,536$ & 0.813839912225434 \\
13 & $34,200,012$ & 0.8196030381092273 & 24 & $18,230,780,418,139$ & 0.8135161559345164 \\
\hline \multicolumn{7}{c}{$* P_{y}(G)$ stands for $\left.\frac{\partial F(G ; y, 1)}{\partial y}\right|_{y=1} \cdot$} \\
\end{tabular}




\section{Concluding Remarks}

In this paper we established the subtree generating functions of the wheel graph $W_{n}$ and "partitions" $K_{1, n}^{j}(1 \leq j \leq n)$, along with other related structures such as the fan graphs. With the subtree generating functions, we are able to study the subtree number and subtree densities of these graphs. From our computational analysis, several theoretical conclusions are drawn, especially on the extremal problems with respect to the subtree number. These results provide a fundamental basis for studying subtree problems of multi-cyclic graphs, chemical compounds, and complex graphs.

As one can see, the computational results in Section 3.2 show interesting observations on the characteristics of these structures. Attempting to verify some of them, especially on those related to the subtree densities, and exploring the BC-subtree number index, Wiener index, Harary index, atom-bond connectivity trends of wheel graph $W_{n}$, "partitions" $K_{1, n}^{j}(1 \leq j \leq n)$, and their generalizations would make very interesting projects for future work. Exploring the novel structural properties from the evolutionary perspective of graphs with more complicated cycle structures, particularly some important nanomaterials such as the pentagonal carbon nanocone, is also a very attractive future topic.

Author Contributions: H.W, Y.Y and A.W contributed to supervision, project administration, and the formal analysis. W.-T.Z and D.-Q.S contributed to the methodology and writing of the original draft preparation. The final draft was written by H.W and Y.Y

Funding: This work is supported by the National Natural Science Foundation of China (grant nos. 61702291, 61772102, 61472058,11531001), the China Postdoctoral Science Foundation (grant no. 2018M632095), the Program for Science \& Technology Innovation Talents in Universities of Henan Province (grant no. 19HASTIT029), the Joint NSFC-ISF Research Program (jointly funded by the National Natural Science Foundation of China and the Israel Science Foundation (No. 11561141001)), the Key Research Project in Universities of Henan Province (grant nos. 19B110011, 19B630015), the Scientific Research Starting Foundation for High-Level Talents of Pingdingshan University (grant no.PXY-BSQD2017006), and the Simons Foundation (grant no. 245307).

Conflicts of Interest: The authors declare no conflict of interest.

\section{Appendix A. Matlab Code, Python Code, And Data}

In what follows, we provide the source codes related to Figure 4. Python code Pythoncode1 (derived from Corollary 2) can output the data files " $n=33 . t x t$ ", " $n=58 . t x t$ ", " $n=75 . t x t$ ", and " $n=80 . t x t$ " by inputting the integer 33, 58, 75, 80, respectively. MATLAB codes MCF1a, MCF1b can draw the Figure $4 \mathrm{a}, \mathrm{b}$, respectively.

Pythoncode1. The Python code for computing the subtree number of $K_{1, n}^{j}(1 \leq j \leq n)$.

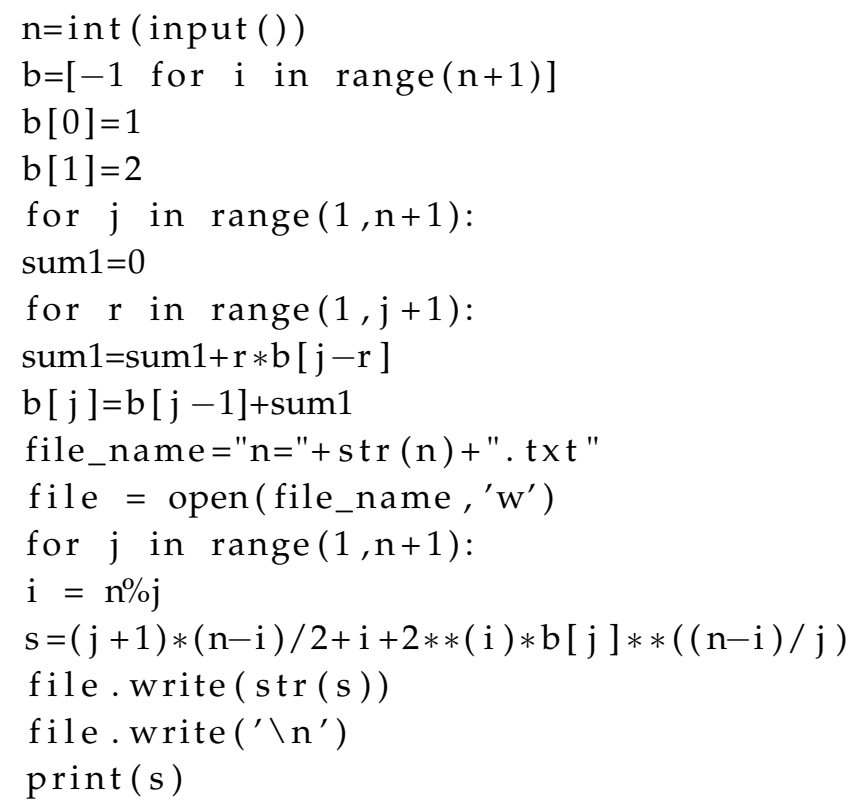


MCF1a. MATLAB source code for Figure 1a.

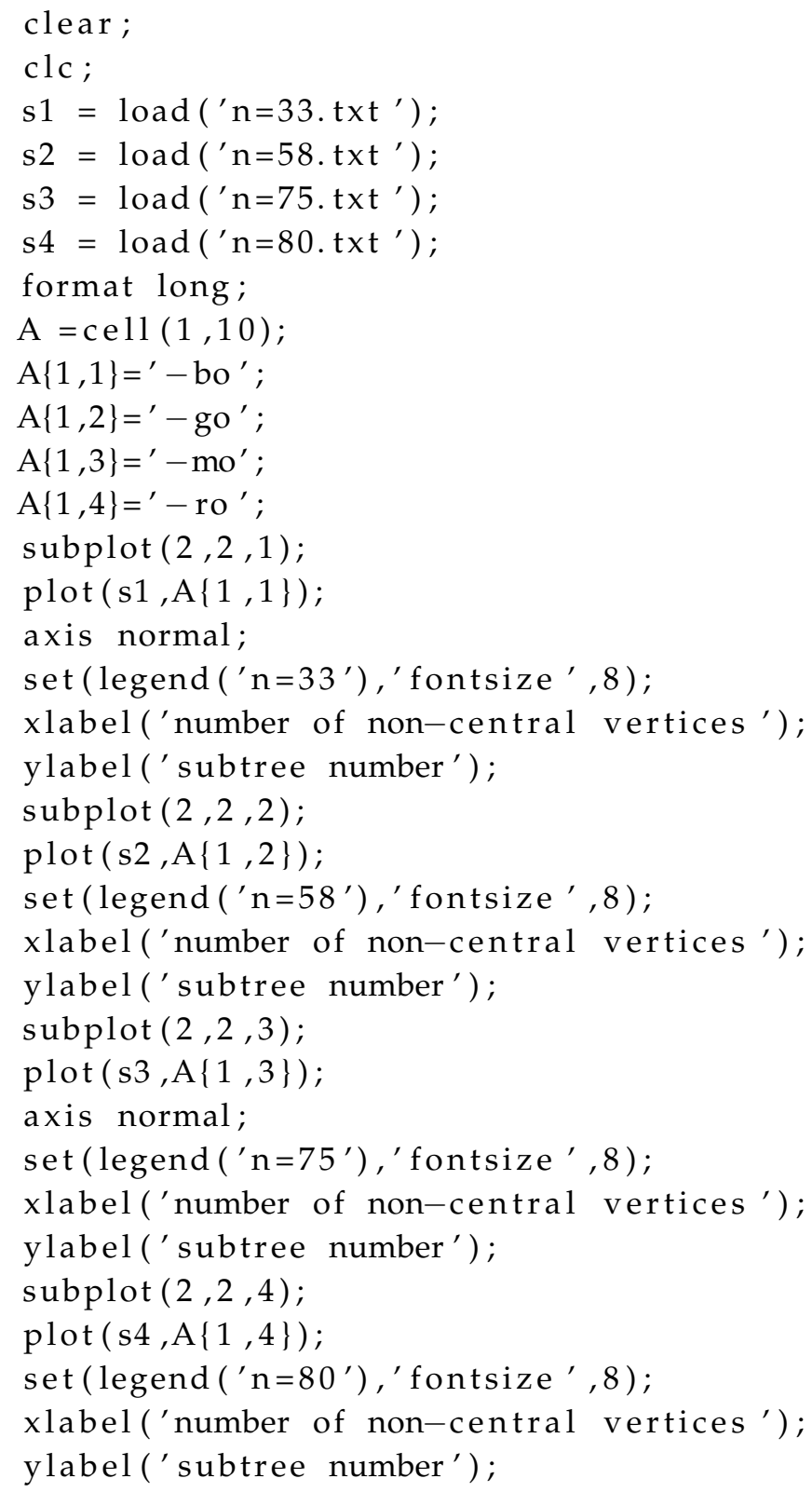

MCF1b: The MATLAB source code for Figure 1b.

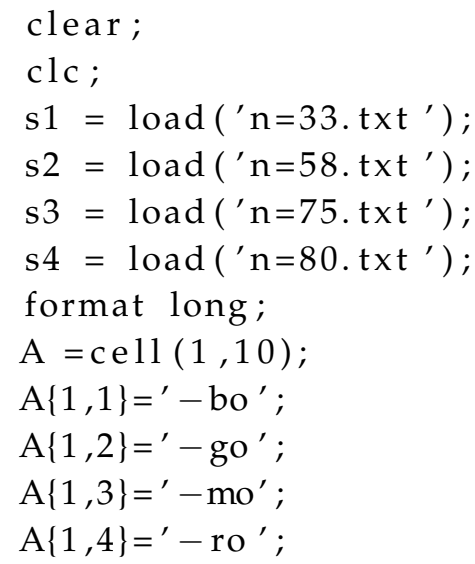




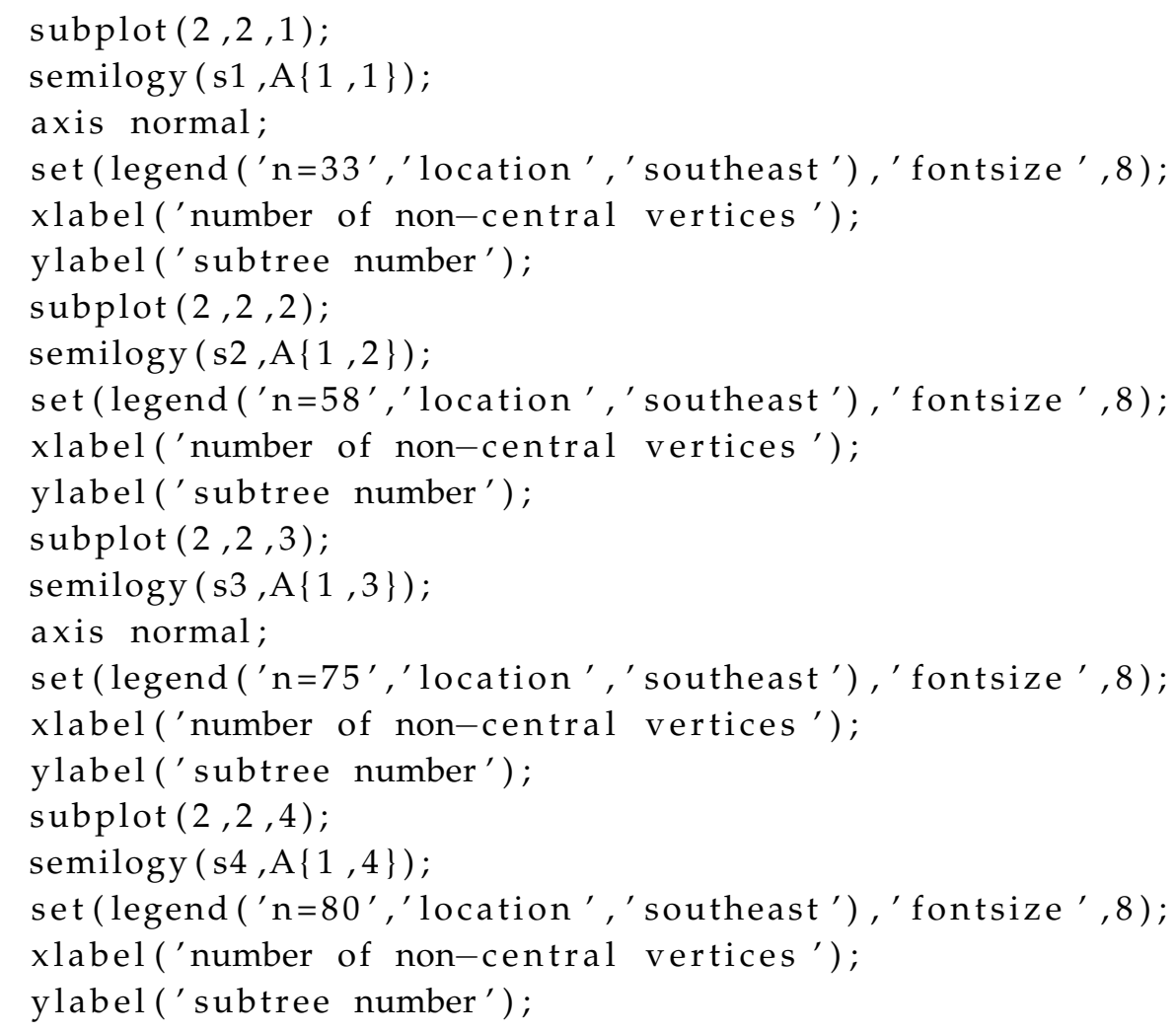

\section{References}

1. Das, K.C. Atom-bond connectivity index of graphs. Discret. Appl. Math. 2010, 158, 1181-1188. [CrossRef]

2. Dobrynin, A.A.; Entringer, R.; Gutman, I. Wiener index of trees: Theory and applications. Acta Appl. Math. 2001, 66, 211-249. [CrossRef]

3. Wiener, H. Structural determination of paraffin boiling points. J. Am. Chem. Soc. 1947, 1, 17-20. [CrossRef]

4. Yan, W.; Yeh, Y. Enumeration of subtrees of trees. Theor. Comput. Sci. 2006, 369, 256-268. [CrossRef]

5. Ingalalli, V.; Ienco, D.; Poncelet, P. Mining frequent subgraphs in multigraphs. Inf. Sci. 2018, 451, 50-66. [CrossRef]

6. Tilk, C.; Irnich, S. Combined column-and-row-generation for the optimal communication spanning tree problem. Comput. Oper. Res. 2018, 93, 113-122. [CrossRef]

7. Zetina, C.A.; Contreras, I.; Fernández, E.; Luna-Mota, C. Solving the optimum communication spanning tree problem. Eur. J. Oper. Res. 2019, 273, 108-117. [CrossRef]

8. Chechik, S.; Emek, Y.; Patt-Shamir, B.; Peleg, D. Sparse reliable graph backbones. Inf. Comput. 2012, 210, 31-39. [CrossRef]

9. Xiao, Y.; Zhao, H.; Liu, Z.; Mao, Y. Trees with large numbers of subtrees. Int. J. Comput. Math. 2017, 94, $372-385$. [CrossRef]

10. Knudsen, B. Optimal multiple parsimony alignment with affine gap cost using a phylogenetic tree. Algorithms Bioinform. Lect. Notes Comput. Sci. 2003, 2812, 433-446.

11. Wagner, S.G. Correlation of graph-theoretical indices. SIAM J. Discret. Math. 2007, 21, 33-46. [CrossRef]

12. Székely, L.; Wang, H. On subtrees of trees. Adv. Appl. Math. 2005, 34, 138-155.

13. Székely, L.; Wang, H. Binary trees with the largest number of subtrees. Discret. Appl. Math. 2007, 155, $374-385$.

14. Zhang, X.; Zhang, X.; Gray, D.; Wang, H. The number of subtrees of trees with given degree sequence. J. Graph Theory 2013, 73, 280-295. [CrossRef]

15. Wang, H. The extremal values of the Wiener index of a tree with given degree sequence. Discret. Appl. Math. 2008, 156, 2647-2654. [CrossRef]

16. Deng, H. Wiener indices of spiro and polyphenyl hexagonal chains. Math. Comput. Model. 2012, 55, 634-644. [CrossRef] 
17. Yang, Y.; Liu, H.; Wang, H.; Fu, H. Subtrees of spiro and polyphenyl hexagonal chains. Appl. Math. Comput. 2015, 268, 547-560. [CrossRef]

18. Czabarka, É.; Székely, L.A.; Wagner, S. On the number of nonisomorphic subtrees of a tree. J. Graph Theory 2018, 87, 89-95. [CrossRef]

19. Zhang, X.; Zhang, X. The Minimal Number of Subtrees with a Given Degree Sequence. Graphs Comb. 2015, 31, 309-318. [CrossRef]

20. Jamison, R.E. On the average number of nodes in a subtree of a tree. J. Comb. Theory Ser. B 1983, 35, $207-223$. [CrossRef]

21. Vince, A.; Wang, H. The average order of a subtree of a tree. J. Comb. Theory Ser. B 2010, 100, 161-170. [CrossRef]

22. Wagner, S.; Wang, H. On the Local and Global Means of Subtree Orders. J. Graph Theory 2016, 81, $154-166$. [CrossRef]

23. Nakayama, T.; Fujiwara, Y. BCT Representation of Chemical Structures. J. Chem. Inf. Comput. Sci. 1980, 20, 23-28. [CrossRef]

24. Nakayama, T.; Fujiwara, Y. Computer representation of generic chemical structures by an extended block-cutpoint tree. J. Chem. Inf. Comput. Sci. 1983, 23, 80-87. [CrossRef]

25. Frederickson, G.N.; Hambrusch, S.E. Planar linear arrangements of outerplanar graphs. IEEE Trans. Circuits Syst. 1988, 35, 323-333. [CrossRef]

26. Wada, K.; Luo, Y.; Kawaguchi, K. Optimal fault-tolerant routings for connected graphs. Inf. Process. Lett. 1992, 41, 169-174. [CrossRef]

27. Heath, L.; Pemmaraju, S. Stack and queue layouts of directed acyclic graphs: Part II. SIAM J. Comput. 1999, 28, 1588-1626. [CrossRef]

28. Misiolek, E.; Chen, D.Z. Two flow network simplification algorithms. Inf. Process. Lett. 2006, 97, 197-202. [CrossRef]

29. Fox, D. Block cutpoint decomposition for markovian queueing systems. Appl. Stoch. Model. Data Anal. 1988, 4, 101-114. [CrossRef]

30. Barefoot, C. Block-cutvertex trees and block-cutvertex partitions. Discret. Math. 2002, 256, 35-54. [CrossRef]

31. Mkrtchyan, V. On trees with a maximum proper partial 0-1 coloring containing a maximum matching. Discret. Math. 2006, 306, 456-459. [CrossRef]

32. Yang, Y.; Liu, H.; Wang, H.; Feng, S. On algorithms for enumerating BC-subtrees of unicyclic and edge-disjoint bicyclic graphs. Discret. Appl. Math. 2016, 203, 184-203. [CrossRef]

33. Yang, Y.; Liu, H.; Wang, H.; Makeig, S. Enumeration of BC-subtrees of trees. Theor. Comput. Sci. 2015, 580, 59-74. [CrossRef]

34. Yang, Y.; Liu, H.; Wang, H.; Deng, A.; Magnant, C. On Algorithms for Enumerating Subtrees of Hexagonal and Phenylene Chains. Comput. J. 2017, 60, 690-710. [CrossRef]

35. Chin, A.J.; Gordon, G.; MacPhee, K.J.; Vincent, C. Subtrees of graphs. J. Graph Theory 2018, 89, 413-438. [CrossRef]

36. Cahit, I. Spiral chains: A new proof of the four color theorem. arXiv 2004, arXiv: 0408247.

37. Yang, Z.; Liu, Y.; Li, X.Y. Beyond trilateration: On the localizability of wireless ad hoc networks. IEEE/ACM Trans. Netw. (ToN) 2010, 18, 1806-1814. [CrossRef]

38. Allen, B.; Lippner, G.; Chen, Y.T.; Fotouhi, B.; Momeni, N.; Yau, S.T.; Nowak, M.A. Evolutionary dynamics on any population structure. Nature 2017, 544, 227. [CrossRef]

39. Haslegrave, J. Extremal results on average subtree density of series-reduced trees. J. Comb. Theory Ser. B 2014, 107, 26-41. [CrossRef]

(C) 2019 by the authors. Licensee MDPI, Basel, Switzerland. This article is an open access article distributed under the terms and conditions of the Creative Commons Attribution (CC BY) license (http://creativecommons.org/licenses/by/4.0/). 